This item was submitted to Loughborough's Research Repository by the author.

Items in Figshare are protected by copyright, with all rights reserved, unless otherwise indicated.

\title{
Influence of topography of nanofibrous scaffolds on functionality of engineered neural tissue
}

PLEASE CITE THE PUBLISHED VERSION

https://doi.org/10.1039/c7tb02969a

\section{PUBLISHER}

(c) The Royal Society of Chemistry

\section{VERSION}

AM (Accepted Manuscript)

\section{PUBLISHER STATEMENT}

This work is made available according to the conditions of the Creative Commons Attribution-NonCommercialNoDerivatives 4.0 International (CC BY-NC-ND 4.0) licence. Full details of this licence are available at: https://creativecommons.org/licenses/by-nc-nd/4.0/

\section{LICENCE}

CC BY-NC-ND 4.0

\section{REPOSITORY RECORD}

Hajiali, Hadi, Andrea Contestabile, Elisa Mele, and Athanassia Athanassiou. 2018. "Influence of Topography of Nanofibrous Scaffolds on Functionality of Engineered Neural Tissue". figshare.

https://hdl.handle.net/2134/33198. 


\title{
Influence of topography of nanofibrous scaffolds on functionality of engineered neural tissue
}

Received 00th January 20xx, Accepted 00th January 20xx

DOI: $10.1039 / x 0 x \times 00000 x$

www.rsc.org/

\begin{abstract}
H. Hajiali ${ }^{a, b, *}$, A. Contestabile ${ }^{c}$, E. Mele ${ }^{d}$, A. Athanassiou ${ }^{a, *}$
Properly engineered scaffolds combined with functional neurons can be instrumental for the effective repair of the neural tissue. In particular, it is essential to investigate how three-dimensional (3D) systems and topographical features can impact on neuronal activity to obtain engineered functional neural tissues. In this study, polyphenylene sulfone (PPSu) scaffolds constituted by randomly distributed or aligned electrospun nanofibers were fabricated to evaluate the neural activity in $3 \mathrm{D}$ culture environments for the first time. The obtained results demonstrated that the nanofibers can successfully support the adhesion and growth of neural stem cells (NSCs) and enhance neuronal differentiation compared to 2D substrates. In addition, NSCs could spread and migrate along the aligned fibers. The percentage of active NSC-derived neurons and the overall network activity in the fibrous substrates were also remarkably enhanced. Finally, the data of neuronal activity showed not only that the neurons cultured on the nanofibers are part of a functional network, but also that their activity increases, and the direction of neural signals can be controlled in the aligned 3D scaffolds.
\end{abstract}

\section{Introduction}

Stroke, neurodegenerative diseases, traumatic brain and spinal cord injury affect millions of patients worldwide ${ }^{1,2}$. Indeed, the regeneration of injured nervous tissues is still an unmet clinical need. The combination of molecular biology ${ }^{3}$ with material science ${ }^{2,4}$ and bio-nanotechnology ${ }^{5}$ may represent a new tool to develop effective neural therapeutic strategies. In particular, several studies on the regeneration of the central nervous system have demonstrated the importance of using both neural stem cells (NSCs) and engineered polymer scaffolds ${ }^{2,6}$. In fact, expandable sources of neurons and properly engineered scaffolds, which can provide neural differentiation to a specific lineage, can be instrumental for the effective repair of the neural tissue. Cells show different reactions based on various microenvironments, and scaffolds can provide threedimensional (3D) culturing systems to mimic the microenvironments of the extracellular matrix (ECM) of human tissues ${ }^{7-9}$.

Because cells interact with their surroundings at the micrometer and nanometer scale, many studies have shown that the topography of scaffolds plays a very important role in controlling and regulating cells in regenerative medicine ${ }^{10,11}$. The fabrication of scaffolds made of aligned electrospun

\footnotetext{
a. Smart Materials, Istituto Italiano di Tecnologia, via Morego 30, 16163 Genoa, Italy.

b. DIBRIS, University of Genoa, via Opera Pia 13, 16145, Genoa, Italy.

Department of Neuroscience and Brain Technologies, Istituto Italiano di

Tecnologia (IIT), via Morego 30, 16163 Genoa, Italy.

d. Department of Materials, Loughborough University, Loughborough, LE11 3TU, United Kingdom.

*Corresponding Authors: hadi.hajiali@edu.unige.it; athanassia.athanassiou@iit.it. Electronic Supplementary Information (ESI) available: [details of any supplementary information available should be included here]. See DOI: 10.1039/x0xx00000x
}

nanofibers can potentially have several beneficial effects on artificial neural tissues, such as control over neurite extension and replication of neuroanatomical pathways ${ }^{12}$. Therefore, scaffolds composed of highly oriented nanofibers are potential tools for restructuring the neural circuitry of the nervous system. Different 3D culture systems and electrospun scaffolds (random and aligned nanofibres) have been shown to support the growth and differentiation of NSCs ${ }^{13}, 14 .{ }^{12}$, ${ }^{15}$. Recently, various synthetic and natural polymers have been applied in neural tissue engineering 2,13,16. Among these, polyaromatic polymers, such as polypyrrole and polyaniline, have been used in many studies ${ }^{16,17}$. Here, polyphenylene sulfone (PPSu) was selected from the same category of polymers (polyaromatic polymers) and its properties were investigated for neural tissue engineering. PPSu scaffolds constituted by randomly distributed or aligned nanofibers were fabricated and used to control NSCs migration, neurite extension and differentiation. Additionally, we observed that NSCs were able to migrate inside the electrospun mats, as they do in a 3D environment. This allowed us to investigate the role played by both nanofibrous architecture and topographical features on the activity of neuronal cells, compared to conventional 2D culture systems. To the best of our knowledge this type of study has not been reported so far. The activity of NSC-derived neurons and primary neuronal cultures in the scaffolds was measured by calcium imaging, in order to assess the potentiality of the nanofibrous scaffolds in rebuilding damaged circuits in the nervous system.

\section{Results and discussion}

\section{Scaffold Characterization}


PPSu can be dissolved in few solvents, including $\mathrm{N}$-methyl pyrrolidinone (NMP) ${ }^{18}$. However, the high boiling point of NMP $\left(202^{\circ} \mathrm{C}\right)$ poses limitations in the fabrication of PPSu fibers by electrospinning. The slow evaporation of NMP delays the solidification of the liquid solution during the extrusion process, and therefore, fibrous membranes do not form. In this study, PPSu was dissolved in a mixture of toluene:Trifluoroacetic acid (TFA) at 60:40 volume ratio (Table S1). PPSu was successfully electrospun and nanofibers free from beads and defects were

a
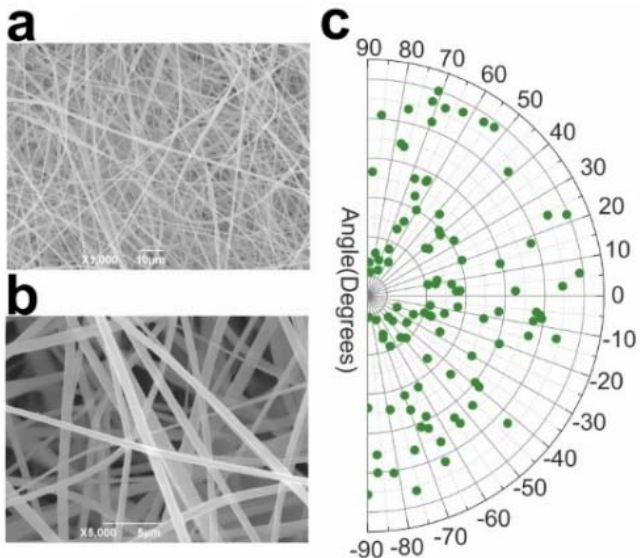

\section{g}

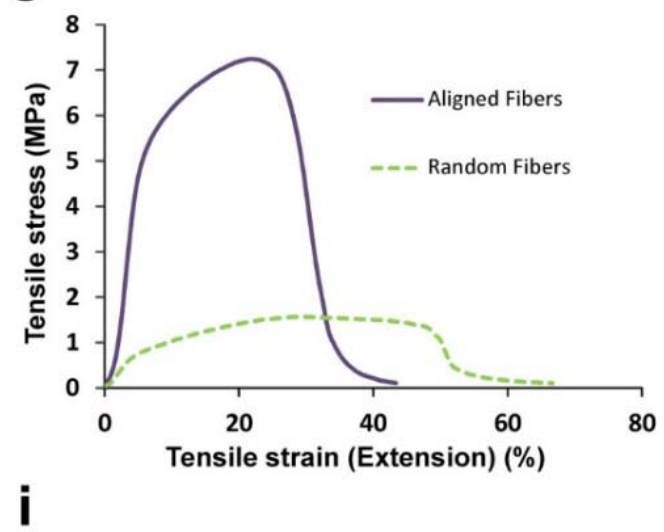

b, c) and aligned (d,

f) PPSu electrospun nanofibers, respectively. $\mathrm{g}, \mathrm{h}, \mathrm{i}, \mathrm{j}, \mathrm{l}$ Mechanical properties of PPSu fibers g) Stress-strain curves of random and aligned fibers, h) Young's Modulus of random and aligned fibers, Ultimate tensile strength of random and aligned fibers, j) Tensile strain of random and aligned fibers at maximum load. previous studies, in order to enhance NSCs spreading, migration and differentiation. We also prepared highly aligned nanofibers, as shown Figure $1 \mathrm{~d}, 1 \mathrm{e}$, and $1 \mathrm{f}$. More than $90 \%$ of the aligned fibers were distributed within an angle ranging from $-10^{\circ}$ to $10^{\circ}$ with respect to the rotating direction of the collector. This is indicative of highly aligned fibers.

The mechanical properties of random and aligned mats were analysed by tensile tests (performed along the fibres direction of the aligned scaffolds). Stress-strain curves of the random and

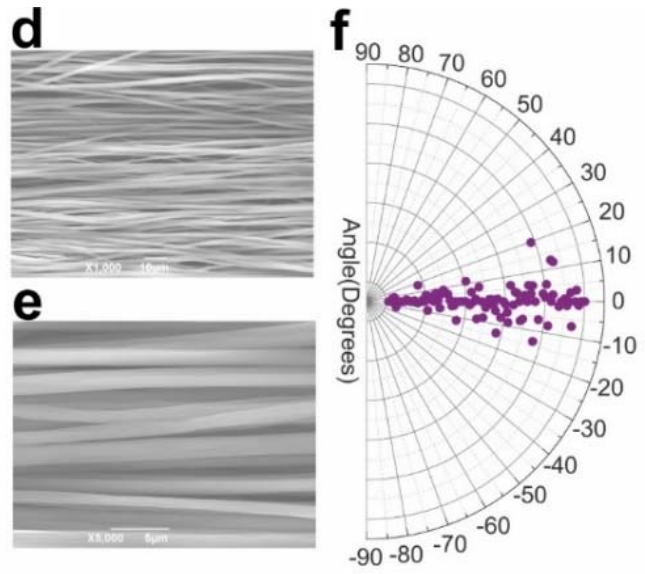

h
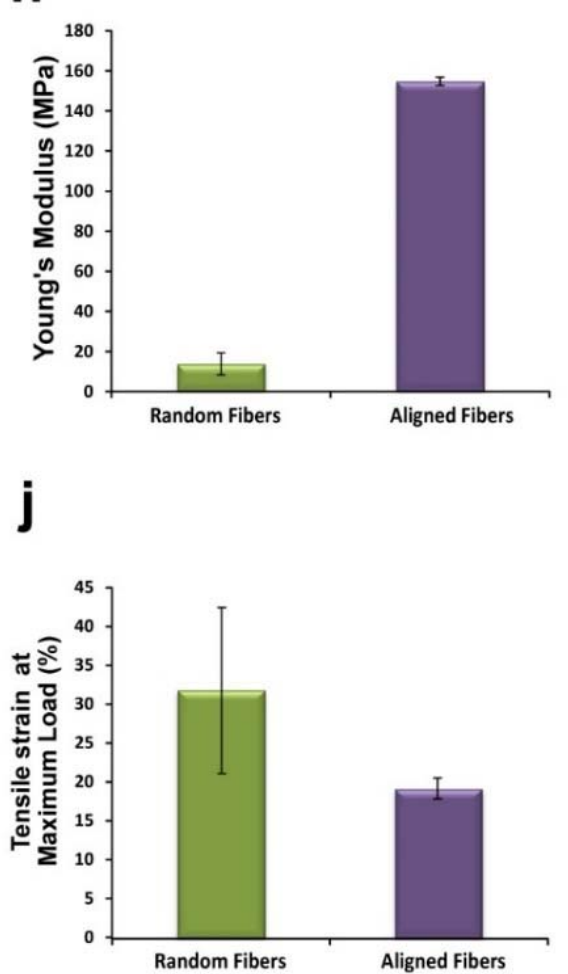

formed (Figure 1). The fibers exhibited homogenous shape and regular morphology, with an average diameter of $735 \mathrm{~nm}$. It has been demonstrated that fiber diameter impacts on the adhesion, proliferation, migration, and differentiation of NSCs 19, 20. A previous work has demonstrated that NSCs show an increase in neuronal differentiation on larger fibers (diameter of $749 \mathrm{~nm}$ ) compared to narrower fibers (diameter of $283 \mathrm{~nm}$ ) 19. Therefore, we optimized the size of PPSu fibers according to aligned fibres, their Young's modulus, tensile strength and elongation at break are shown in Figure $1 \mathrm{~g}-1 \mathrm{j}$. The electrospun membranes made of random and aligned fibers showed a tensile strength of $(1.6 \pm 0.3) \mathrm{MPa}$ and $(6.7 \pm 0.6) \mathrm{MPa}$, respectively (Figure $1 \mathrm{i}$ ). The tensile strength of the aligned fibers increased about four-fold when compared to that of random fiber mats. Moreover, the aligned fibers were characterized by Young's modulus of $(154.7 \pm 2.1) \mathrm{MPa}$ (10 times higher than 
Young's modulus of random fibers) and elongation at break of $19 \%$. Thus, the parallel alignment of fibers significantly increased tensile strength and Young's modulus of the structures in the direction of fibers, while the elongation at break did not change significantly.

Similar to the obtained results, Alvarez et al. reported that random poly-lactic acid nanofibers had a remarkably lower strength and Young's modulus compared to the aligned fibers, as determined in a uniaxial tensile assay parallel to the direction of the nanofibers ${ }^{21}$. Furthermore, Kijenska et al. fabricated random and aligned Poly (L-lactic acid)-co-poly(e-

a

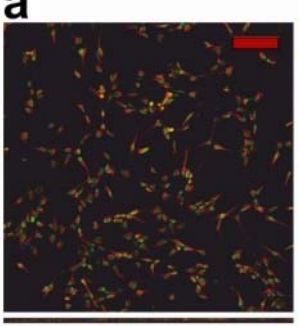

c

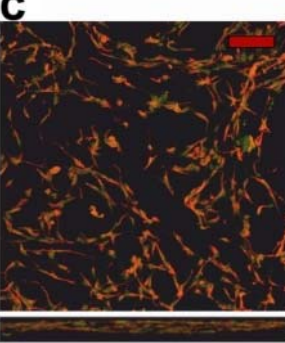

image of neural stem cells (NSCs) expressed the markers Nestin (red) and SOX2(green) on a) glass, b) PPSu film, c) random fibers, d) aligned fibers; Scale bar, $100 \mu \mathrm{m}$; e) NCS viability on different substrates by MTT assay $\left(^{*}: p<0.05\right)$; and $\mathrm{f}, \mathrm{g}$ ) SEM image of neural stem cells on f) random fibers, g) aligned fibers. $h, i, j$, Timelapse imaging of GFP expressed-NSCs on $\mathrm{h}$ ) control, i) random fibers, j) aligned fibers, k) NSCs velocity in different samples, and I) NSC velocity in $X$ and $Y$ direction (*: $p<0.05)$.

h

i

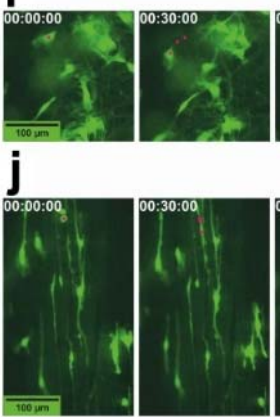

b

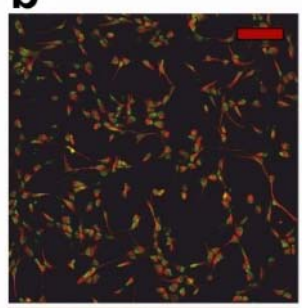

d

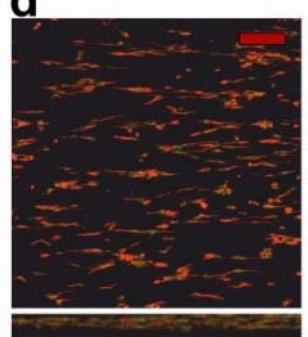

caprolactone)/collagen nanofibers and investigated their mechanical properties. Their results also demonstrated that aligned fibers displayed higher average tensile strength and Young's modulus than the random nanofibers, but their elongation at break was similar to those of the random fibers 22 . In our study, both random and aligned fibers have sufficient strength in order to be handled for biomedical applications.

The wettability of PPSU films and fibers was investigated because of its importance on cell attachments. ${ }^{23}$ Water contact angle measurements demonstrated that PPSu films were quite hydrophilic with WCA of $(65 \pm 4)^{\circ}$, whereas PPSu fibers were
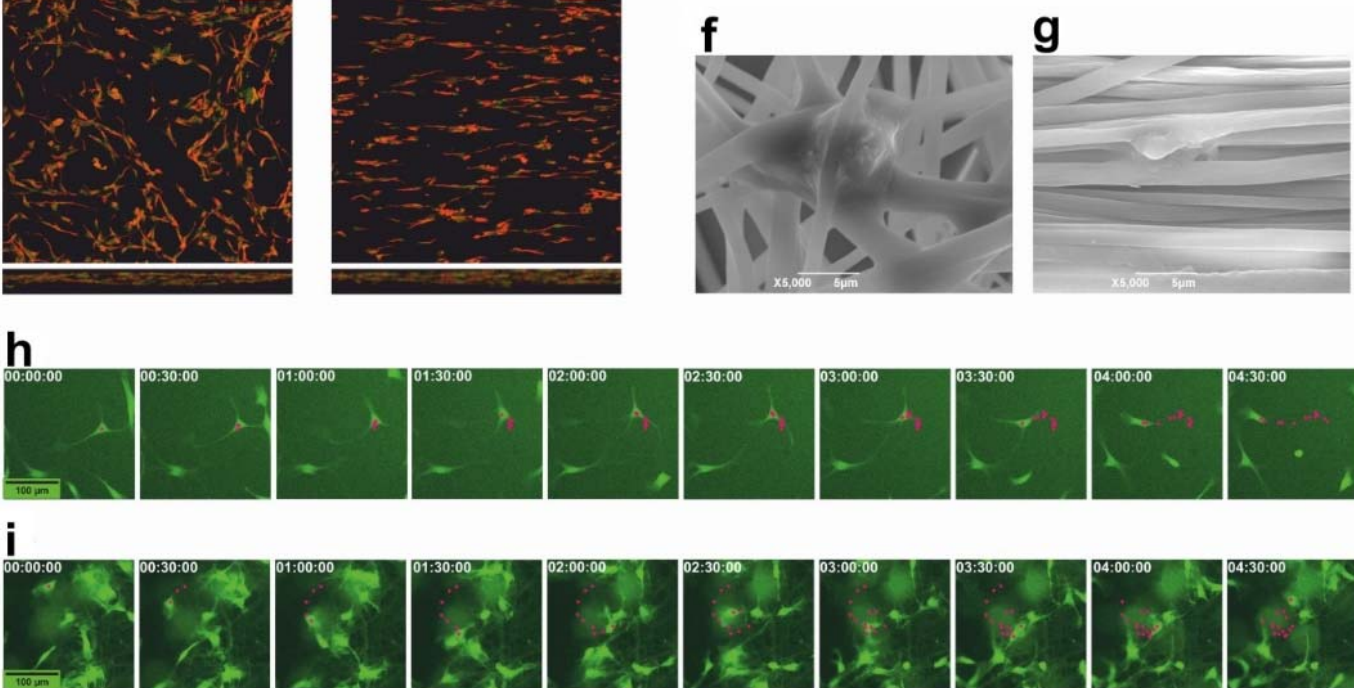

g
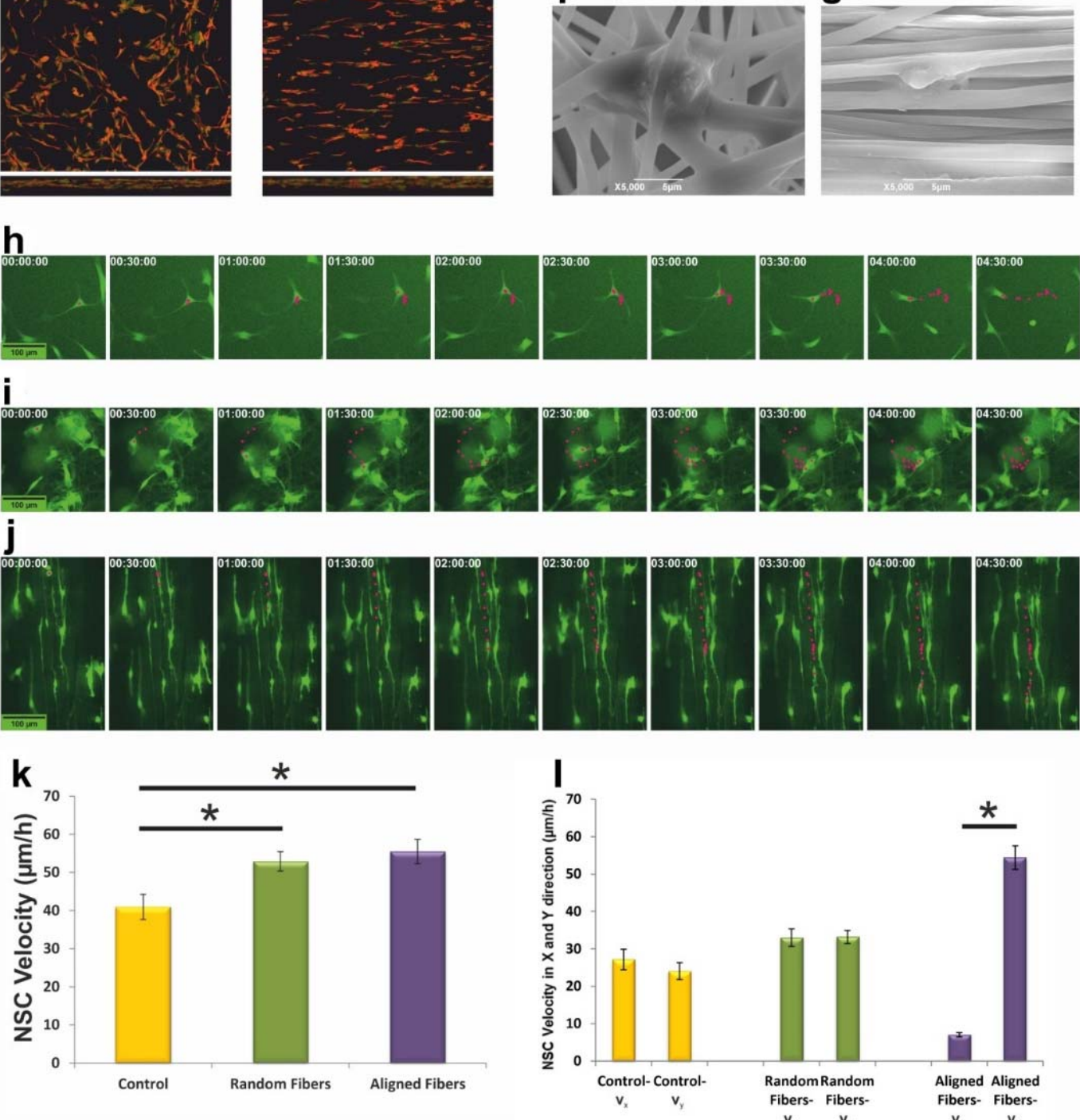
hydrophobic with an apparent WCA of $(138 \pm 6)^{\circ}$, most likely because of the air trapped within the voids of the fibrous scaffolds. As previous studies showed, hydrophilic substrates are more favourable for cell adhesion on their surface, with respect to hydrophobic ones. ${ }^{23,} 24$ On the other hand, surface geometry is another important factor influencing cell attachment and behaviour, 23, 24 and in our study, the cells adhesion and extension onto the PPSu films and fibers seem to be more influenced by the surface structure rather than the apparent wettability.

\section{NSCs Proliferation}

The PPSu films and fibers were sterilized by autoclaving before seeding the cells. Among different types of polymers (polycaprolactone, polylactic acid, polyethylene, polypropylene, polyamide, polysulfone, and natural polymers), PPSU is the best polymer for resistance in the autoclaving process because of its high glass-transition temperature $\left(220^{\circ} \mathrm{C}\right)$ which show an increase in utilizing PPSu for medical applications 29,30 .

Furthermore, as can be seen in Figure 2e, a significant improvement of NSCs proliferation $(p<0.05)$ was observed on the PPSu fibers compared to PPSu films, due to the fibrous architecture. The enhancement of NSCs viability on the aligned and random fibers compared to PPSu film and glass coverslip proves that not only PPSu is an acceptable biocompatible material for neural tissue engineering, but also the topography of culturing scaffolds can impact on NSCs growth and viability, regardless of the chemical composition of the substrate.

In previous studies, it was demonstrated that nanofibrous scaffolds with proper topography can provide a favourable 3D environment for the proliferation of different type of cells ${ }^{31}$. Like in our study, no statistical difference was found in neonatal mouse cerebellum C17.2 stem cells viability between the aligned and random fibers $(p>0.05)^{32}$. Our results prove that electrospun PPSu scaffolds support the growing of NSCs cells a

Figure 3. Confocal image of differentiated neural stem cells (s) expressed the marker Tuj1(red) on a) glass, b) PPSu film, c) random fibers, $d$ aligned fibers; Scale bar, $50 \mu \mathrm{m}$; e) Percentage of cells positive for Tuj1 marker, f) Percentage of active cells in timelapse calcium imaging (*: $p<$ $0.05)$.

C b

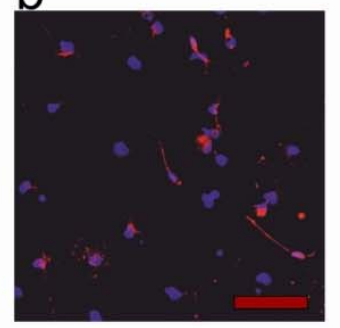

d
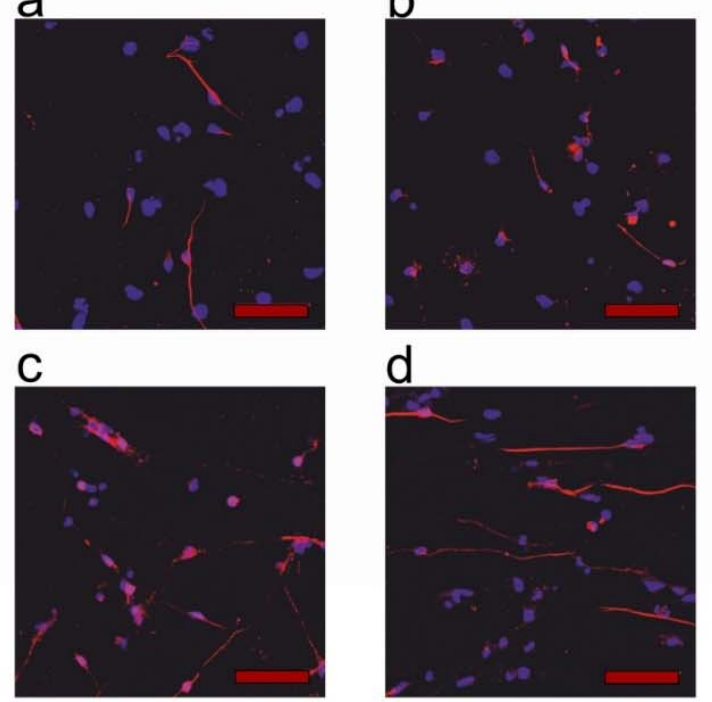

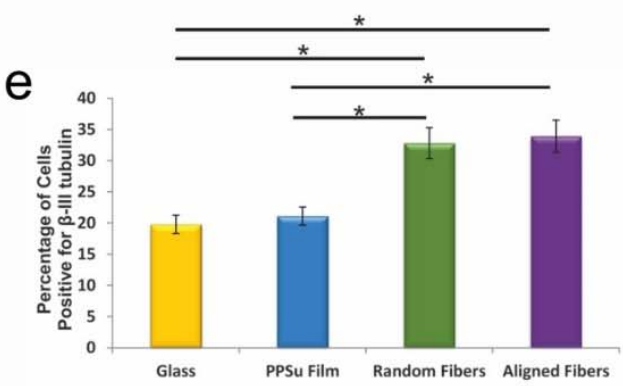

f

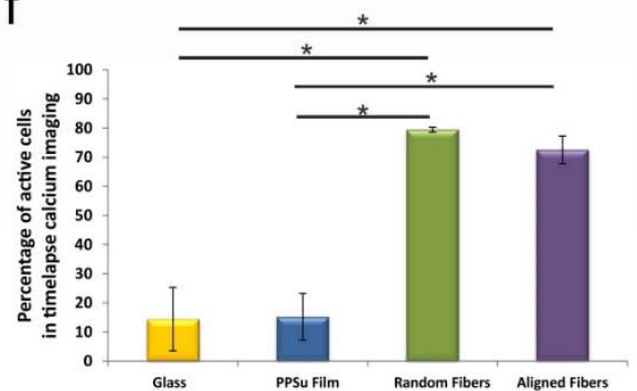

25. We used nestin and Sox2 immunostaining to evaluate cell morphology and arrangement in the scaffold material (Figure 2a,b,c,d). Importantly, NSCs grown on PPSu scaffolds were able to proliferate, migrate and infiltrate inside the voids of the fibers (Figure 2c, 2f). Moreover, they did not alter the expression of the progenitor markers. The cells grown on the aligned fibers were able to elongate following the direction of fibers, creating aligned parallel patterns (Figure $2 \mathrm{~d}$ and $2 \mathrm{~g}$ ). The viability of NSCs and 3 T3 fibroblasts was evaluated by MTT assay, as shown in Figure 2e and Figure S1, respectively. These results demonstrate that the average number of viable cells on the PPSu films and fibers was higher than the number of cells on glass coverslips, which were used as control $(p<0.05)$. We hypothesize that the higher biocompatibility of PPSu was due to the presence of sulfonate groups. In fact, sulfonate groups have shown enhanced cell attachment in other studies 26,27 . In addition, commercial products of PPSu (Radel ${ }^{\circledR}$ R) have been approved by FDA ${ }^{28}$, and there are also some recent patents and they can be employed to interact with NSCs and provide a supportive 3D environment in which the NSCs are enabled to proliferate.

\section{NSCs Migration}


The movement of GFP-expressing NSCs in control, random and aligned fibers is shown in Figure $2 \mathrm{~h}, \mathrm{i}$ and $\mathrm{j}$, respectively, and in Videos S1-S3. The cells on the fibers not only moved as fast as the control but also they penetrated into the fibers and formed the 3D structure. The movement of cells in the $z$ plane and therefore their infiltration inside the electrospun mat was also demonstrated by the change of focus for some cells in the images. We also analyzed the
The increase in the cell migration velocity induced by fibers topography has also an effect in enhancing the penetration of cells into the electrospun mats, resulting in the formation of a 3D cellular network in a short period of time. In Figure $2 \mathrm{l}$, the large difference between $v_{x}$ and $v_{y}$ for the aligned fibers indicates that cell migration is highly governed by the fibers direction and that the speed of the cell migration is maximized

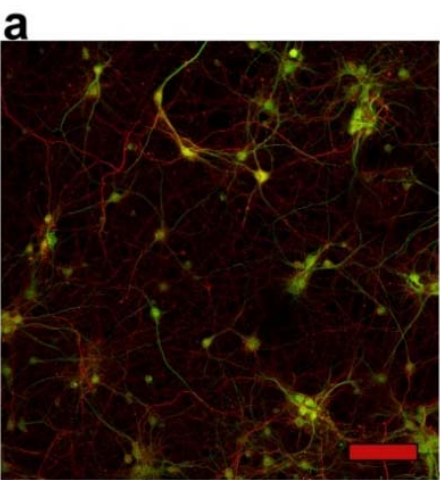

d
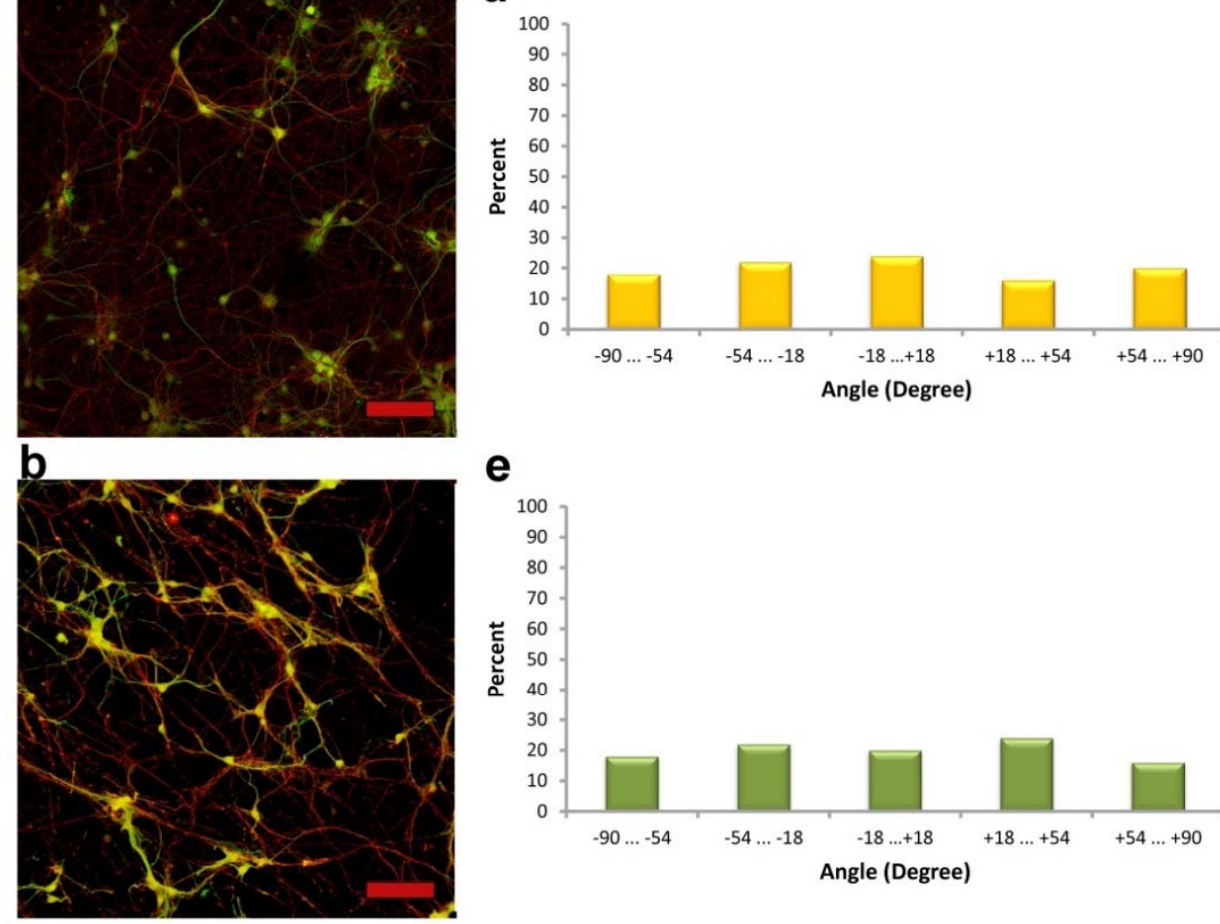

Figure 4. $a, b, c)$ Confocal image of neuronal cells that expressed the markers SMI312(red-for axons) and Map2(green-for dendrites) on a) glass, b) random fibers, and c) aligned fibers; Scale bar, 100 $\mu \mathrm{m}$; d,e, f) Angle distribution of axons of neuronal cells on $d$ ) glass, e) random fibers, and f) aligned fibers.
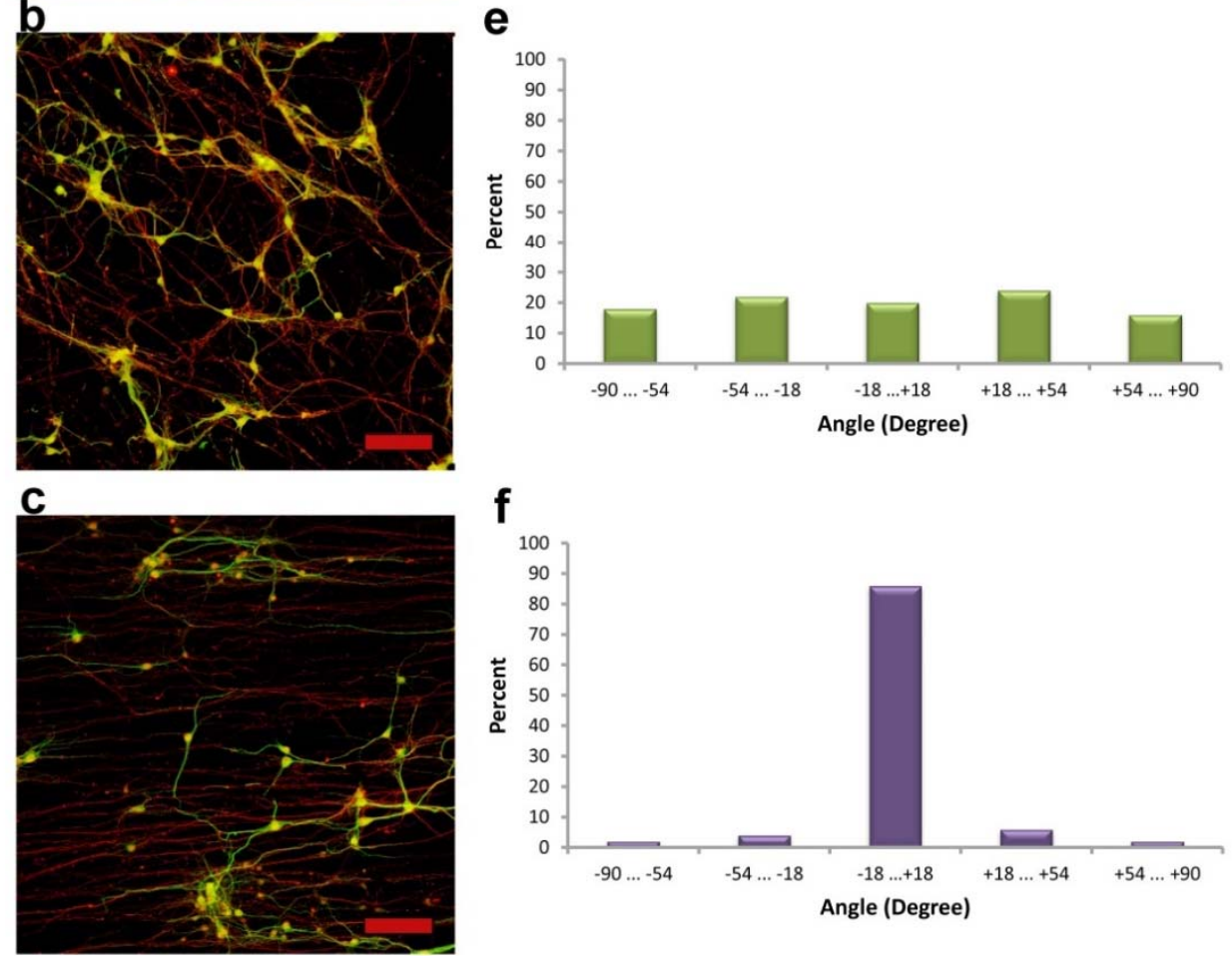

direction of cells movement and found that most of the cells moved along the direction of the fibers. Therefore, the direction of cells migration can be significantly controlled by using aligned nanofibers.

In order to quantitatively analyze the speed and direction of cell migration, the average velocity of NSCs and the directional velocity in $x\left(v_{x}\right)$ and $y$-axis $\left(v_{y}\right)$ were calculated by the Tracker software and the results are shown in Figure $2 \mathrm{k}$ and $2 \mathrm{l}$. The $\mathrm{y}-$ axis was defined in the direction of the aligned fibers for the coordinate systems in the software. The speed of NSCs migration on control samples was $(40 \pm 18) \mu \mathrm{m} / \mathrm{h}$; while the cells moved significantly faster with a speed of $(52 \pm 13) \mu \mathrm{m} / \mathrm{h}$ and $(55 \pm 17) \mu \mathrm{m} / \mathrm{h}$ on the random and aligned fibers, respectively.

towards the direction of fibers.

\section{NSCs Differentiation and Activity}

The differentiation potential of NSCs towards neuronal cells was studied by evaluating the expression of neuronal marker TUJ1 (tubulin $\beta 3$ ) and Doublecortin (DCX). As shown in Figure 3 and Figure S2, the percentage of cells positive for TUJ1 and DCX for random and aligned fibers (3D cultures) was significantly higher than for the control (glass) and PPSu films (2D cultures). These results demonstrate quantitatively that 3D nanofibrous scaffolds can sustain NSCs differentiation. Lee et al. have showed that $3 \mathrm{D}$ culture of stem cells promotes the increased 
expression of neural phenotypes and the secretion of neurotrophic factors when compared to 2D culture ${ }^{33}$. Moreover, it has been demonstrated that the topographic signals at nanoscale from the local stem cell niche can control the behaviours of neural stem cells and regulate stem cell fate $12,15,34$. Our data are consistent with a previous study ${ }^{35}$, where it has been shown that on aligned fibers a higher fraction of cells exhibited markers of neuronal differentiation as compared with
Furthermore, we evaluated the activity of differentiated NSC plated on different samples by time-lapse calcium imaging. Video S4 shows spontaneous calcium transients in differentiated NSCs on different substrates. Interestingly, we found that the percentage of active cells for the 3D fibrous samples was largely greater than for 2D culture system and control (Figure 3f). Carlson et al. have investigated the response
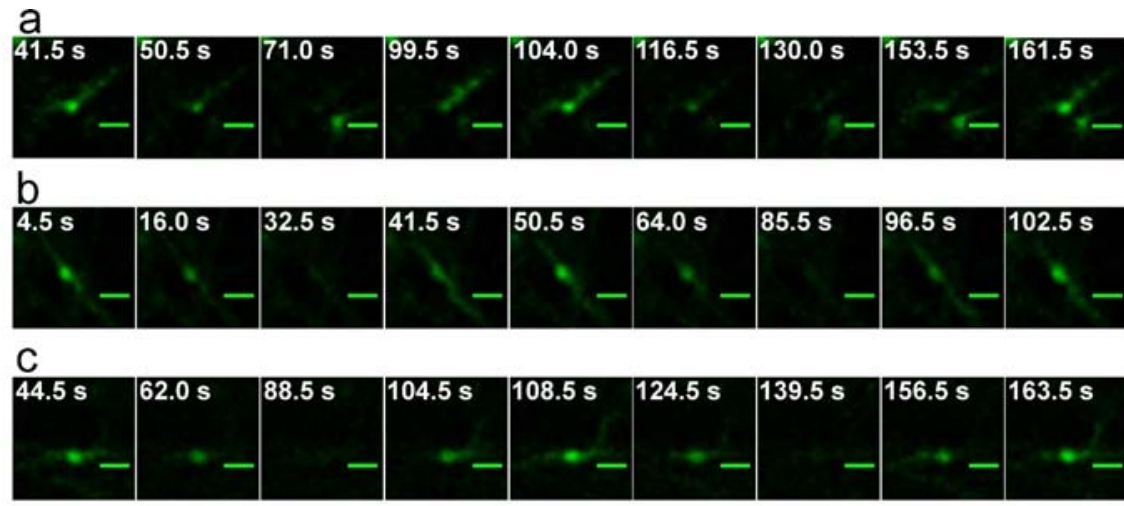
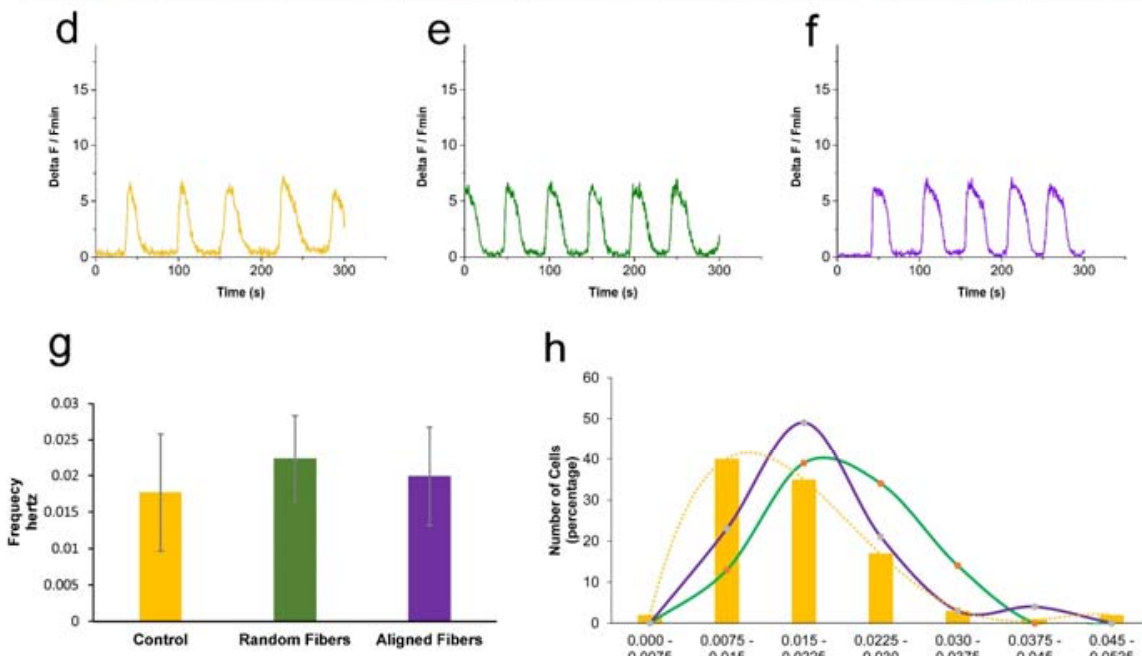

$\mathrm{h}$

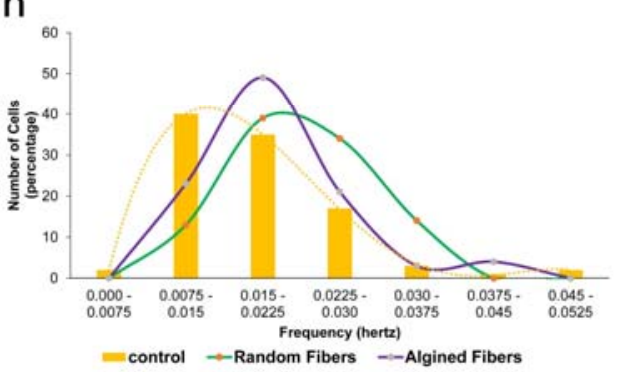

Figure 5. a,b,c) Timelapse calcium imaging of neuron cell (a single cell undergoing a transient increase and decrease in signal; Scale bar, $20 \mu \mathrm{m}$ ) on a) control, b) random fibers, and c) aligned fibers; d,e,f) Normalized intensity trace for a single neuron cell on d) control, e) random fibers, and f) aligned fibers; g) Mean of frequency for cell flashing on different substrates; and h) Distribution of frequency of calcium activity for different samples.

cells on random fibers or unpatterned surfaces after induction of NSCs differentiation with retinoic acid. of induced neuronal cells in 2D and 3D cultures to electrical stimulation, and they have observed that a lower fraction of 
cells in the 3D substrates was active ${ }^{36}$. In contrast, our results show that not only the number of active cells significantly increased in the fibrous substrates but also the frequency of calcium transient of active cells on these scaffolds was remarkably enhanced, as it can be seen in Video S4.

\section{Neuronal Activity}

Finally, we assessed the culture of primary neuronal cells on the 3D fibrous scaffolds. We used immunohistochemistry for the dendritic and axonal markers (Map2 and Smi312R) to evaluate neuronal morphology onto different samples. As shown in Figure $4 a-4 c$ neuronal axons grew and extended following primarily the direction of the fibers. For the aligned fibers, they were able to form a pattern of very long parallel structures (Figure $4 \mathrm{f}$ ). In fact, more than $85 \%$ of axons on aligned fibers showed an angle distribution between $-18^{\circ}$ and $18^{\circ}$, indicating the high potential of our 3D scaffolds for neuronal tissue engineering applications.

Next, we used time-lapse calcium imaging of neuronal cells to investigate the activity of cells cultured onto different samples (Video S5 and S6). Figure 5a-5c show examples of a cell on glass, random and aligned fibers undergoing calcium transient. The data were quantified by normalizing intensity trace of the signal for each neuron cell on the different substrates (Figure $5 d-f$ ). We found that the majority of cells exhibited spontaneous calcium transients (Video S5), suggesting that they were part of an active neuronal network. In addition, a variety of patterns of calcium transients was seen in fibers and control samples (Figure $5 \mathrm{~g}$ ). In a previous study, the monitoring of fluorescence intensity of spontaneous $\mathrm{Ca}^{2+}$ oscillations within the differentiated neural aggregates in culture has also shown a wide range of activity frequencies ${ }^{37}$. Timashev et al. have fabricated scaffolds with a 3D structure, which are composed of two layers of cylinders (height $100 \mathrm{~mm}$, inside diameter $100 \mu \mathrm{m}$, outside diameter $160 \mu \mathrm{m}$ ), and they evaluated the calcium activity of hippocampal cells on their scaffolds and under control conditions (coverslips). In their study, the neurons of dissociated hippocampal cultured on the 3D cylinders scaffolds revealed a decrease in the frequency of $\mathrm{Ca}^{2+}$ oscillations compared to the cells in 2D culture ${ }^{38}$.

By analyzing 100 active cells for each sample, we found a significant difference in the distribution of frequency of calcium activity $(p<0.05)$ (Figure $5 \mathrm{~h}$ ). For the electrospun fibers, the variance of data was reduced and the number of cells with a short period of activity was enhanced compared to the control. This means that a greater number of cells with higher frequency was active onto the fibers. Thus, our results demonstrated an enhanced activity of cells when they were cultured onto the 3D nanofibrous scaffolds, clearly indicating a marked effect of nanofibrous scaffold topography on neuronal activity for the first time. In addition, the data of aligned nanofibers showed a bimodal frequency distribution of calcium activity (Figure $5 \mathrm{~h}$ ). In fact, a second peak at even higher frequency $(0.0375-0.045 \mathrm{~Hz})$ is observed only for the data of aligned nanofibers. This can be due to an optimized connection of a number of cells when they grow on the aligned fibers, that further facilitates the calcium wave movement. Interestingly, Video $\mathrm{S} 6$ shows that calcium waves could move along the direction of fibers, indicating the possible utilization of fibrous scaffolds to control the direction of neural signals.

\section{Experimental}

\section{Materials and cells}

Polyphenylene sulfone (PPSu), Trifluoroacetic acid (TFA), Toluene (TO), poly-D-lysine (PDL), laminin(Lam), Dulbecco's modified Eagle's medium (DMEM), Bovine Calf Serum (BCS), Trypsin-EDTA solution, Penicillin-Streptomycin, Phosphate Buffered Saline (PBS), Triton, Fluoroshield with DAPI, and Thiazolyl Blue Tetrazolium Bromide (MTT) were purchased from Sigma-Aldrich. NSCs were isolated from the hippocampal dentate gyrus (DG) of adult (6-8 weeks) WT mice. Neuronal cells were isolated from the cortex of mice.

\section{Aligned and random nanofibers preparation}

In this paper, we describe a method to produce random and highly aligned PPSu nanofibers using a combination of two solvents, toluene and trifluoroacetic acid (TFA), never used before to dissolve this polymer. PPSu is insoluble in each of these solvents separately, but highly soluble in their blend. The solubility of PPSu was evaluated in diverse blends of toluene, acetone, chloroform, ethanol, and TFA. The only solvent system which could dissolve PPSu was a mixture of toluene and TFA. Different volume concentrations of toluene:TFA, from 0 to 100 $\%$ volume ratio, were prepared, and their ability to dissolve PPSu at various wt\% ratios $(10,15,20,25)$ was investigated at room temperature (Table S1). Our observations demonstrated that PPSu showed the highest solubility in 60:40 volume ratio of toluene:TFA. PPSu was instead insoluble in this system if the concentration of TFA exceeded $60 \%$ by volume.

Solutions of PPSu (25 wt\%) in Toluene:TFA (60:40) were prepared for the electrospinning process. A syringe with a stainless steel 23-gauge needle was filled with the solution and connected to a syringe pump working at a constant flow rate of $1 \mathrm{~mL} /$ hour. A voltage of $15 \mathrm{kV}$ was applied between the needle and an aluminum collector (air gap distance of $15 \mathrm{~cm}$ ) by using a high-voltage power supply. Randomly distributed fibers were collected in a static aluminum foil. A rotating collector with high speed (5000 rpm) was utilized to produce aligned fibers.

\section{Scaffold characterization}

\section{SEM}

The morphology, size, and angle distribution of the electrospun nanofibers were analyzed by scanning electron microscopy (SEM). Prior to imaging, the fibrous mats were covered with 5 $\mathrm{nm}$ of gold, deposited by ion sputtering. The diameter and angle distribution of the fibers were determined by processing the SEM images by Image J program.

\section{Mechanical properties}

The mechanical properties of the electrospun PPSu fiber membranes were measured using a uniaxial testing machine (Instron 3365 dual column) under a cross-head speed of $50 \mathrm{~mm} /$ minute and a gauge length of $25 \mathrm{~mm}$. From the stress- 
strain curves, Young's modulus, tensile strength, and elongation at break were calculated.

\section{Contact Angle}

In order to investigate the wettability of the nanofibers, the water contact angle (WCA) was evaluated by a video-based optical contact angle measuring instrument DataPhysics OCA 20 (Germany). Water droplets of a volume of $10 \mu \mathrm{l}$ were gently placed on the surfaces of the samples and the measurements were conducted on three different sample areas and averaged for each sample.

\section{NSCs Culture}

Mouse NSCs, previously isolated from the adult dentate gyrus of wild-type mice ${ }^{39}$, were cultured in flasks coated with 10 $\mu \mathrm{g} / \mathrm{ml}$ poly-D-lysine (PDL) and $5 \mu \mathrm{g} / \mathrm{ml}$ laminin (Lam). NSCs were grown in Neurobasal medium supplemented with $2 \%(\mathrm{v} / \mathrm{v})$ B27 supplement minus Vitamin A, 1\% (v/v) GlutaMAX, 1\% (v/v) penicillin-streptomycin (all from Invitrogen) and $20 \mathrm{ng} / \mathrm{ml}$ each of bFGF and EGF (Peprotech). Cells were passaged at $70-80 \%$ confluence using Accutase (Invitrogen) and re-plated at $10^{4}$ cells $/ \mathrm{cm}^{2}$. The proliferation of cells plated on scaffolds or glass coverslips was evaluated 24 hours after plating by a colorimetric MTT assay. GFP-expressing NSCs were obtained by infection with a lentivirus expressing Enhanced Green Fluorescent protein as previously described ${ }^{40}$.

\section{NSCs Differentiation}

For differentiation experiments, NSCs were seeded in the same medium (NSCs culture medium) as above but without EGF. After $24 \mathrm{~h}$, the medium was replaced with differentiation medium (Neurobasal medium supplemented with $2 \%(\mathrm{v} / \mathrm{v})$ standard B27 supplement, 1\% (v/v) GlutaMAX and penicillin-streptomycin) supplemented with $5 \mathrm{ng} / \mathrm{ml}$ of bFGF. After additional 48 hours, the medium was replaced with differentiation medium containing $1 \mathrm{ng} / \mathrm{ml}$ of bFGF. Finally, after additional 48 hours, the medium was replaced with differentiation medium without growth factors.

\section{NSCs Migration assay}

The GFP-expressing NSCs were seeded on samples in the conditions which are described above, and $24 \mathrm{~h}$ later, imaged for additional 24 hours with a Nikon Time-Lapse Microscopy equipped with an environmental chamber (Okolab. Temperature: 37 C, Humidity (RH): $95 \%$, CO2 Concentration: 5 $\%$, Flow: $0.70 \mathrm{l} / \mathrm{min}$ ). The interval between successive images was $15 \mathrm{~min}$. Cell movements were analyzed by Tracker software (http://www.opensourcephysics.org).

\section{Neuronal Culturing}

Mouse cortical neurons were isolated as previously described 40. The Cells were seeded on samples coated with $100 \mu \mathrm{g} / \mathrm{ml}$ poly-L-lysine (PLL) in Neurobasal medium supplemented with $2 \%(\mathrm{v} / \mathrm{v})$ B27 supplement, 1\% (v/v) GlutaMAX and penicillinstreptomycin solution. Two weeks later, the samples were fixed and processed for immunocytochemistry.

\section{Calcium Imaging}

For calcium imaging, cells were loaded with $2.5 \mu \mathrm{M}$ Fluo-4 calcium indicator (Invitrogen) in Tyrode's solution (140 mM $\mathrm{NaCl}, 4 \mathrm{mM} \mathrm{KCl}, 2 \mathrm{mM} \mathrm{CaCl} 2,2 \mathrm{mM} \mathrm{MgCl} 2,10 \mathrm{mM}$ HEPES, 10 $\mathrm{mM}$ Glucose) for 15 minutes at room temperature in the dark. Imaging was performed on a Nikon A1 scanning confocal microscope. Amplitude and frequency of calcium events were measured on the region of interest (ROI) centered on cell bodies with NIS-Elements software (Nikon).

\section{Immunocytochemistry}

Samples were fixed in 4 wt\% PFA in PBS for $20 \mathrm{~min}$. Samples were then washed in PBS $(3 \times 5 \mathrm{~min})$ and preamiblized with $0.1 \%$ $(\mathrm{v} / \mathrm{v})$ Tirion in PBS (PBST). After blocking with $5 \%(\mathrm{v} / \mathrm{v})$ normal goat serum (NGS; Jackson ImmunoResearch Inc) in PBST for 1 hour, the samples were incubated with the following primary antibodies in blocking solution overnight at $4{ }^{\circ} \mathrm{C}$ : mouse antitubulin $\beta 3$ (TUJ1; 1:400; Covance), rabbit anti-DCX (1:200; abcam), mouse anti-Nestin (1:200; Millipore), rabbit anti-SOX2 (1:200; Millipore), rabbit anti-MAP2 (1:500; Covance), mouse anti-Smi312R (1:500; Covance). After washing in PBST $(3 \times$ $5 \mathrm{~min})$, samples were incubated with fluorophore-conjugated (Alexa Fluor 488, Alexa Fluor 568, and Alexa Fluor 647) goat secondary antibodies (1:500; Invitrogen) for 2 hours at room temperature. The samples were washed again in PBST $(3 \times 5$ $\mathrm{min}$ ) and finally mounted with Pro-long Gold mounting medium (Invitrogen) containing DAPI nuclear dye.

\section{Statistical analysis}

All data were presented as means \pm SD. One-way ANOVA was used to evaluate statistical significance, followed by Bonferroni's post-hoc test. OriginPro was used for all statistical analysis. Kolmogorov-Smirnov combined with Sidak multiple comparison test was used to compare the distribution of data obtained by calcium imaging. The $p$ values less than 0.05 were considered significant.

\section{Conclusions}

In the present study, random and aligned PPSu nanofibers with an optimized diameter were successfully electrospun using toluene:TFA mixture as solvent. To our knowledge, this is the first work that investigates the effect of 3D nanofibrous scaffolds with different topography (including aligned and random fibers) on the activity of neurons and NSC-derived neurons. In particular, 3D PPSu nanofibrous scaffolds were produced and applied for neural tissue engineering, focusing on their possible functional roles in neural regeneration. The obtained results demonstrated that the 3D PPSu nanofibers can successfully support the adhesion and growth of NSCs and enhance neuronal differentiation compared to 2D substrates. In addition, NSCs could spread and migrate along the aligned fibers. The percentage of active NSC-derived neurons and their frequency of activity in the fibrous substrates were remarkably enhanced. Moreover, nanofibers also supported the growth and activity of primary neuronal cells. The immunocytochemical analysis of axon and dendrite growth of neurons exhibited a great dependence on the fibers' arrangement. The pattern of 
parallel axon growth was obtained on the aligned nanofibers, and this can be utilized for guiding the neurite outgrowth with an appropriate function. Furthermore, the data of calcium imaging showed not only that the neuronal cells are part of a functional network but also that their activity can increase, and the movement of neural signals can be controlled when they grow in the 3D nanofibrous scaffolds. To conclude, our results demonstrate that the developed 3D scaffolds have the ideal properties and topographical features for their use in neural tissue engineering.

\section{References}

1. V. Chandra, R. Pandav and R. Laxminarayan, in Disease Control Priorities in Developing Countries. 2nd edition, eds. D. Jamison, J. Breman and A. Measham, World Bank, Washington (DC), 2006, pp. 627-643.

2. R. Y. Tam, T. Fuehrmann, N. Mitrousis and M. S. Shoichet, Neuropsychopharmacology, 2014, 39, 169-188.

3. E. M. Tanaka and P. Ferretti, Nat Rev Neurosci, 2009, 10, 713723.

4. G. Orive, E. Anitua, J. L. Pedraz and D. F. Emerich, Nat Rev Neurosci, 2009, 10, 682-692.

5. G. A. Silva, Nat Rev Neurosci, 2006, 7, 65-74.

6. L. Binan, C. Tendey, G. De Crescenzo, R. El Ayoubi, A. Ajji and M. Jolicoeur, Biomaterials, 2014, 35, 664-674.

7. H. Hajiali, J. A. Heredia-Guerrero, I. Liakos, A. Athanassiou and E. Mele, Biomacromolecules, 2015, 16, 936-943.

8. M. P. Lutolf and J. A. Hubbell, Nat Biotech, 2005, 23, 47-55.

9. H. Hajiali, S. Shahgasempour, M. R. Naimi-Jamal and H. Peirovi, International journal of nanomedicine, 2011, 6, 21332141.

10. C. Czeisler, A. Short, T. Nelson, P. Gygli, C. Ortiz, F. P. Catacutan, B. Stocker, J. Cronin, J. Lannutti and J. Winter, Journal of Comparative Neurology, 2016, 524, 3485-3502.

11. H. Miyoshi and T. Adachi, Tissue Engineering Part B: Reviews, 2014, 20, 609-627.

12. W. Chen, Y. Shao, X. Li, G. Zhao and J. Fu, Nano Today, 2014, 9, 759-784.

13. A. Subramanian, U. M. Krishnan and S. Sethuraman, Journal of biomedical science, 2009, 16, 108.

14. L. G. Zhang and D. L. Kaplan, Neural Engineering: From Advanced Biomaterials to 3D Fabrication Techniques, Springer International Publishing, 2016.

15. J. Xie, M. R. MacEwan, A. G. Schwartz and Y. Xia, Nanoscale, 2010, 2, 35-44.

16. Y.-S. Lee and T. Livingston Arinzeh, Polymers, 2011, 3, 413.

17. L. Ghasemi-Mobarakeh, M. P. Prabhakaran, M. Morshed, M. H. Nasr-Esfahani, H. Baharvand, S. Kiani, S. S. Al-Deyab and S. Ramakrishna, Journal of tissue engineering and regenerative medicine, 2011, 5, e17-35.

18. B. Ellis and R. Smith, Polymers: A Property Database, Second Edition, CRC Press, 2008.

19. G. T. Christopherson, H. Song and H. Q. Mao, Biomaterials, 2009, 30, 556-564.

20. R. J. Wade and J. A. Burdick, Nano Today, 2014, 9, 722-742.
21. Z. Alvarez, O. Castano, A. A. Castells, M. A. Mateos-Timoneda, J. A. Planell, E. Engel and S. Alcantara, Biomaterials, 2014, 35, 4769-4781.

22. E. Kijenska, M. P. Prabhakaran, W. Swieszkowski, K. J. Kurzydlowski and S. Ramakrishna, Journal of biomedical materials research. Part B, Applied biomaterials, 2012, 100, 1093-1102.

23. H.-I. Chang and Y. Wang, in Regenerative medicine and tissue engineering-cells and biomaterials, InTech, 2011.

24. D. P. Dowling, I. S. Miller, M. Ardhaoui and W. M. Gallagher, Journal of biomaterials applications, 2011, 26, 327-347.

25. B. Ellis and R. Smith, Polymers: a property database, CRC Press, 2009.

26. A. S. G. CURTIS, J. V. FORRESTER, C. McINNES and F. LAWRIE, The Journal of Cell Biology, 1983, 97, 1500-1506.

27. H. Felgueiras and V. Migonney, Materials science \& engineering. C, Materials for biological applications, 2014, 39, 196-202.

28.

http://www.accessdata.fda.gov/cdrh docs/pdf11/K113039.p df, 2012.

29. , 2013.

30. , 2010 .

31. M. Borjigin, B. Strouse, R. A. Niamat, P. Bialk, C. Eskridge, J. Xie and E. B. Kmiec, Molecular Therapy-Nucleic Acids, 2012, 1, e59. 32. L. He, S. Liao, D. Quan, K. Ma, C. Chan, S. Ramakrishna and J. Lu, Acta Biomater, 2010, 6, 2960-2969.

33. J. H. Lee, J.-Y. Lee, S. H. Yang, E.-J. Lee and H.-W. Kim, Acta biomaterialia, 2014, 10, 4425-4436.

34. M. Alessandri, G. Lizzo, C. Gualandi, C. Mangano, A. Giuliani, M. Focarete and L. Calza, Matrix Biology, 2014, 33, 68-76.

35. S. H. Lim, X. Y. Liu, H. Song, K. J. Yarema and H.-Q. Mao, Biomaterials, 2010, 31, 9031-9039.

36. A. L. Carlson, N. K. Bennett, N. L. Francis, A. Halikere, S. Clarke, J. C. Moore, R. P. Hart, K. Paradiso, M. Wernig, J. Kohn, Z. P. Pang and P. V. Moghe, Nature Communications, 2016, 7, 10862.

37. E. J. Gualda, D. Simão, C. Pinto, P. M. Alves and C. Brito, Frontiers in Cellular Neuroscience, 2014, 8.

38. P. Timashev, M. Vedunova, D. Guseva, E. Ponimaskin, A. Deiwick, T. Mishchenko, E. Mitroshina, A. Koroleva, A. Pimashkin and I. Mukhina, Biomedical Physics \& Engineering Express, 2016, 2, 035001.

39. A. Contestabile, B. Greco, D. Ghezzi, V. Tucci, F. Benfenati and L. Gasparini, The Journal of clinical investigation, 2013, 123, 348361.

40. D. Pozzi, G. Lignani, E. Ferrea, A. Contestabile, F. Paonessa, R. D'alessandro, P. Lippiello, D. Boido, A. Fassio and J. Meldolesi, The EMBO journal, 2013, 32, 2994-3007.

1 